\title{
DERIVATIONS AND IDENTITIES FOR KRAVCHUK POLYNOMIALS
}

\author{
LEONID BEDRATYUK
}

\begin{abstract}
We introduce the notion of Kravchuk derivations of the polynomial algebra. We prove that any element of the kernel of the derivation gives a polynomial identity satisfied by the Kravchuk polynomials. Also, we prove that any kernel element of the basic Weitzenbök derivations yields a polynomial identity satisfied by the Kravchuk polynomials. We describe the corresponding intertwining maps.
\end{abstract}

Dedicated to the 120th Anniversary of Mykhailo Kravchuk

\section{INTRODUCTION}

The binary Kravchuk polynomials $K_{n}(x, a)$ are defined by the following explicit formula

$$
K_{n}(x, a):=\sum_{i=0}^{n}(-1)^{i}\left(\begin{array}{l}
x \\
i
\end{array}\right)\left(\begin{array}{l}
a-x \\
n-i
\end{array}\right), n=0,1, \ldots,
$$

with the ordinary generating function

$$
\sum_{i=0}^{\infty} K_{i}(x, a) z^{i}=(1+z)^{a}\left(\frac{1-z}{1+z}\right)^{x} .
$$

We are interested in finding polynomial identities satisfied by the polynomials. In other words, polynomials $P\left(x_{0}, x_{1}, \ldots, x_{n}\right)$ in $n+1$ variables such that

$$
P\left(K_{0}(x, a), K_{1}(x, a), \ldots, K_{n}(x, a)\right)=\varphi_{1}(a) \text { or } P\left(K_{0}(x, a), K_{1}(x, a), \ldots, K_{n}(x, a)\right)=\varphi_{2}(x),
$$

where $\varphi_{1}$, and $\varphi_{2}$ are polynomials of one variable. We provide a method for finding such identities which is based on the simple observation: if

$$
\frac{d}{d x} P\left(K_{0}(x, a), K_{1}(x, a), \ldots, K_{n}(x, a)\right)=0 \text { or } \frac{d}{d a} P\left(K_{0}(x, a), K_{1}(x, a), \ldots, K_{n}(x, a)\right)=0,
$$

then $P\left(K_{0}(x, a), K_{1}(x, a), \ldots, K_{n}(x, a)\right)$ is a function only of $a$ or $P\left(K_{0}(x, a), \ldots, K_{n}(x, a)\right)$ is a function of only one variable $x$, i.e., it is a polynomial identity.

On the other hand, rewrite this derivative in the form

$$
\begin{gathered}
\frac{d}{d x} P\left(K_{0}(x, a), K_{1}(x, a), \ldots, K_{n}(x, a)\right)= \\
=\left.\frac{\partial}{\partial x_{0}} P\left(x_{0}, x_{1}, \ldots, x_{n}\right)\right|_{\left\{x_{i}=F_{i}(x)\right\}} \frac{d}{d x} K_{0}(x, a)+\cdots+\left.\frac{\partial}{\partial x_{n}} P\left(x_{0}, \ldots, x_{n}\right)\right|_{\left\{x_{i}=F_{i}(x)\right\}} \frac{d}{d x} K_{n}(x, a) .
\end{gathered}
$$

Suppose that the derivative $\frac{d}{d x} K_{i}(x, a)$ can be expressed as a polynomial of the Kravchuk polynomials, i.e., $\frac{d}{d x} K_{i}(x, a)=f_{i}\left(K_{0}(x, a), \ldots, K_{n}(x, a)\right), i \leq n$, and $f_{i}\left(x_{0}, x_{1}, \ldots, x_{n}\right) \in$ $\mathbb{Q}\left[x_{0}, x_{1}, \ldots, x_{n}\right], 0 \leq i \leq n$. Then 


$$
\begin{gathered}
\frac{d}{d x} P\left(K_{0}(x, a), K_{1}(x, a), \ldots, K_{n}(x, a)\right)= \\
=\left.\left(\frac{\partial}{\partial x_{0}} P\left(x_{0}, x_{1}, \ldots, x_{n}\right) D\left(x_{0}\right)+\cdots+\frac{\partial}{\partial x_{n}} P\left(x_{0}, x_{1}, \ldots, x_{n}\right) D\left(x_{n}\right)\right)\right|_{\left\{x_{i}=F_{i}(x)\right\}}= \\
=\left.\mathcal{D}_{\mathcal{K}_{1}}\left(P\left(x_{0}, x_{1}, \ldots, x_{n}\right)\right)\right|_{\left\{x_{i}=F_{i}(x)\right\}},
\end{gathered}
$$

where the differential operator $\mathcal{D}_{\mathcal{K}_{1}}$ on $\mathbb{Q}\left[x_{0}, x_{1}, \ldots, x_{n}\right]$ is defined by $\mathcal{D}_{\mathcal{K}_{1}}\left(x_{i}\right):=f_{i}\left(x_{0}, x_{1}, \ldots, x_{n}\right)$. It is clear that if $\mathcal{D}_{\mathcal{K}_{1}}\left(P\left(x_{0}, x_{1}, \ldots, x_{n}\right)\right)=0$ then $\frac{d}{d x} P\left(K_{0}(x, a), K_{1}(x, a), \ldots, K_{n}(x, a)\right)=$ 0 . Thus, any non-trivial polynomial $P\left(x_{0}, x_{1}, \ldots, x_{n}\right)$, which belongs to the kernel of $\mathcal{D}_{\mathcal{K}_{1}}$, i.e., the following holds $\mathcal{D}_{\mathcal{K}_{1}}\left(P\left(x_{0}, x_{1}, \ldots, x_{n}\right)\right)=0$, defines a polynomial identity of the form $P\left(K_{0}(x, a), \ldots, K_{n}(x, a)\right)=\varphi(a)$ for some polynomial $\varphi$.

Analogously, suppose that

$$
\frac{d}{d a} K_{i}(x, a)=g_{i}\left(K_{0}(x, a), \ldots, K_{n}(x, a)\right), g_{i} \in \mathbb{Q}\left[x_{0}, x_{1}, \ldots, x_{n}\right],
$$

and define the differential operator $\mathcal{D}_{\mathcal{K}_{2}}$ by $\mathcal{D}_{\mathcal{K}_{2}}\left(x_{i}\right):=g_{i}\left(x_{0}, x_{1}, \ldots, x_{n}\right)$. Then any non-trivial polynomial $P\left(x_{0}, x_{1}, \ldots, x_{n}\right)$, which belongs to the kernel of $\mathcal{D}_{\mathcal{K}_{2}}$ defines a polynomial identity of the form $P\left(K_{0}(x, a), \ldots, K_{n}(x, a)\right)=\varphi(x)$ for some polynomial $\varphi$. If a polynomial $P\left(x_{0}, x_{1}, \ldots, x_{n}\right)$ belongs to the intersection of kernels of the operators $\mathcal{D}_{\mathcal{K}_{1}}$ and $\mathcal{D}_{\mathcal{K}_{2}}$ then it defines an identity $P\left(K_{0}(x, a), \ldots, K_{n}(x, a)\right)=$ const.

For instance, it is easily verified that

$$
\begin{aligned}
\frac{d}{d x} K_{0}(x, a) & =0 & \frac{d}{d a} K_{0}(x, a) & =0, \\
\frac{d}{d x} K_{1}(x, a) & =-2 K_{0}(x, a) & \frac{d}{d a} K_{1}(x, a) & =K_{0}(x, a), \\
\frac{d}{d x} K_{2}(x, a) & =-2 K_{1}(x, a) & \frac{d}{d a} K_{2}(x, a) & =-\frac{1}{2} K_{0}(x, a)+K_{1}(x, a) .
\end{aligned}
$$

Then define the differential operators $\mathcal{D}_{\mathcal{K}_{1}}, \mathcal{D}_{\mathcal{K}_{2}}$ by

$$
\begin{array}{ll}
\mathcal{D}_{\mathcal{K}_{1}}\left(x_{0}\right)=0 & \mathcal{D}_{\mathcal{K}_{2}}\left(x_{0}\right)=0 \\
\mathcal{D}_{\mathcal{K}_{1}}\left(x_{1}\right)=x_{0} & \mathcal{D}_{\mathcal{K}_{2}}\left(x_{1}\right)=x_{0}, \\
& \\
\mathcal{D}_{\mathcal{K}_{1}}\left(x_{2}\right)=x_{1} & \mathcal{D}_{\mathcal{K}_{2}}\left(x_{2}\right)=-\frac{1}{2} x_{0}+x_{1} .
\end{array}
$$

For the polynomial $x_{1}^{2}-2 x_{2} x_{0}$ we have $D_{x}\left(x_{1}^{2}-2 x_{2} x_{0}\right)=0$, thus the expression $K_{1}(x, a)^{2}-$ $2 K_{2}(x, a) K_{0}(x, a)$ is a function of $a$ only. The substitution $K_{n}(x, a)$ of $x_{n}$ gives

$$
K_{1}(x, a)^{2}-2 K_{2}(x, a) K_{0}(x, a)=a .
$$

In the same manner, the polynomial $x_{0} x_{1}-x_{1}^{2}+2 x_{2} x_{0}$ lies in the kernel of the operator $\mathcal{D}_{\mathcal{K}_{2}}$. Therefore the expression $K_{0}(x, a) K_{1}(x, a)-K_{1}(x, a)^{2}+2 K_{2}(x, a) K_{0}(x, a)$ depends on $x$ only. In fact, after simplification we obtain

$K_{0}(x, a) K_{1}(x, a)-K_{1}(x, a)^{2}+2 K_{2}(x, a) K_{0}(x, a)=-2 x-(-2 x+a)^{2}+4 x^{2}-4 a x+a^{2}=-2 x$. 
A similar problem was solved by the present author for the Appellpolynomials. In the paper 11 was proved that any non-trivial element of kernel of the differential operator

$$
\mathcal{D}=x_{0} \frac{\partial}{\partial x_{1}}+2 x_{1} \frac{\partial}{\partial x_{2}}+\cdots+n x_{n-1} \frac{\partial}{\partial x_{n}},
$$

gives some polynomial identity for the Appellpolynomials. Recall that polynomials $\left\{A_{n}(x)\right\}$, $\operatorname{deg}\left(A_{n}(x)\right)=n$ is called the Appellpolynomials if

$$
A_{n}^{\prime}(x)=n A_{n-1}(x), n=0,1,2, \ldots
$$

The operator $\mathcal{D}\left(x_{i}\right)=n x_{i-1}$ is called the basic Weitzenböck derivation and its cernel is isomorphic to some algebra of $S L_{2}$-invariants. In the present paper we show how the kernel elements of the derivation $\mathcal{D}$ can be used to find a polynomials identities for the Kravchuk polynomials. A multiplicative linear map $\psi_{A K_{1}}$ is called $\left(\mathcal{D}, \mathcal{D}_{\mathcal{K}_{1}}\right)$-interwining map if the following condition holds: $\psi_{A K_{1}} \mathcal{D}=\mathcal{D}_{\mathcal{K}_{1}} \psi_{A K_{1}}$. Any such map induces an isomorphism from ker $\mathcal{D}$ to ker $\mathcal{D}_{\mathcal{K}_{1}}$. For instance, the discriminant of the polynomial (in the variables $X, Y$ )

$$
x_{0} X^{3}+3 x_{1} X^{2} Y+3 x_{2} X Y^{2}+x_{3} Y^{3},
$$

equals

$$
\left|\begin{array}{ccccc}
x_{0} & 3 x_{1} & 3 x_{2} & x_{3} & 0 \\
0 & x_{0} & 3 x_{1} & 3 x_{2} & x_{3} \\
3 x_{0} & 6 x_{1} & 3 x_{2} & 0 & 0 \\
0 & 3 x_{0} & 6 x_{1} & 3 x_{2} & 0 \\
0 & 0 & 3 x_{0} & 6 x_{1} & 3 x_{2}
\end{array}\right|=27\left(6 x_{0} x_{3} x_{2} x_{1}+3 x_{1}{ }^{2} x_{2}{ }^{2}-4 x_{1}{ }^{3} x_{3}-4 x_{2}{ }^{3} x_{0}-x_{0}{ }^{2} x_{3}{ }^{2}\right)
$$

and lies in the kernel of the operator $\mathcal{D}$. It is well known result of the classical invariant theory. It is easily checked that the linear map defined by

$$
\begin{aligned}
& \psi_{A K_{1}}\left(x_{0}\right)=x_{0}, \psi_{A K_{1}}\left(x_{1}\right)=x_{1}, \\
& \psi_{A K_{1}}\left(x_{2}\right)=2 x_{2}, \psi_{A K_{1}}\left(x_{3}\right)=-2 x_{1}+6 x_{3},
\end{aligned}
$$

commutes with the operators $\mathcal{D}$ and $\mathcal{D}_{\mathcal{K}_{1}}$. Therefore the element

$$
\begin{gathered}
\left|\begin{array}{ccccc}
\psi_{A K_{1}}\left(x_{0}\right) & 3 \psi_{A K_{1}}\left(x_{1}\right) & 3 \psi_{A K_{1}}\left(x_{2}\right) & \psi_{A K_{1}}\left(x_{3}\right) & 0 \\
0 & \psi_{A K_{1}}\left(x_{0}\right) & 3 \psi_{A K_{1}}\left(x_{1}\right) & 3 \psi_{A K_{1}}\left(x_{2}\right) & \psi_{A K_{1}}\left(x_{3}\right) \\
3 \psi_{A K_{1}}\left(x_{0}\right) & 6 \psi_{A K_{1}}\left(x_{1}\right) & 3 \psi_{A K_{1}}\left(x_{2}\right) & 0 & 0 \\
0 & 3 \psi_{A K_{1}}\left(x_{0}\right) & 6 \psi_{A K_{1}}\left(x_{1}\right) & 3 \psi_{A K_{1}}\left(x_{2}\right) & 0 \\
0 & 0 & 3 \psi_{A K_{1}}\left(x_{0}\right) & 6 \psi_{A K_{1}}\left(x_{1}\right) & 3 \psi_{A K_{1}}\left(x_{2}\right)
\end{array}\right|= \\
=\left|\begin{array}{ccccc}
x_{0} & 3 x_{1} & 6 x_{2} & -2 x_{1}+6 x_{3} & 0 \\
0 & x_{0} & 3 x_{1} & 6 x_{2} & -2 x_{1}+6 x_{3} \\
3 x_{0} & 6 x_{1} & 6 x_{2} & 0 & 0 \\
0 & 3 x_{0} & 6 x_{1} & 6 x_{2} & 0 \\
0 & 0 & 3 x_{0} & 6 x_{1} & 6 x_{2}
\end{array}\right|,
\end{gathered}
$$

lies in the kernel of the operator $\mathcal{D}_{\mathcal{K}_{1}}$ and defines the following identity for the Kravchuk polynomials: 


$$
\begin{array}{|ccccc}
K_{0}(x, a) & 3 K_{1}(x, a) & 6 K_{2}(x, a) & -2 K_{1}(x, a)+6 K_{3}(x, a) & 0 \\
0 & K_{0}(x, a) & 3 K_{1}(x, a) & 6 K_{2}(x, a) & -2 K_{1}(x, a)+6 K_{3}(x, a) \\
3 K_{0}(x, a) & 6 K_{1}(x, a) & 6 K_{2}(x, a) & 0 & 0 \\
0 & 3 K_{0}(x, a) & 6 K_{1}(x, a) & 6 K_{2}(x, a) & 0 \\
0 & 0 & 3 K_{0}(x, a) & 6 K_{1}(x, a) & 6 K_{2}(x, a) \\
& & & \\
& & & \\
& & &
\end{array}
$$

In the paper we present methods of the theory of locally nilpotent derivation to find polynomial identities for the Kravchuk polynomials.

In section 2 ,we give a brief introduction to the theory of locally nilpotent derivations. Also, we introduce the notion of the Kravchuk derivations and find its kernels. In this way we obtain some polynomials identities for the Kravchuk polynomials.

In the section 3 we find a $\left(\mathcal{D}, \mathcal{D}_{\mathcal{K}_{1}}\right)$-intertwining map and a $\left(\mathcal{D}, \mathcal{D}_{\mathcal{K}_{2}}\right)$-intertwining map.

\section{KRAVCHUK DERIVATIONS}

2.1. Derivations and its kernels. Let $\mathbb{Q}\left[x_{0}, x_{1}, x_{2}, \ldots, x_{n}\right]$ be the polynomial algebra in $n+1$ variables $x_{0}, x_{1}, x_{2}, \ldots, x_{n}$ over $\mathbb{Q}$. Recall that a derivation of the polynomial algebra $\mathbb{Q}\left[x_{0}, x_{1}, x_{2}, \ldots, x_{n}\right]$ is a linear map $D$ satisfying the Leibniz rule:

$$
D\left(x_{1} x_{2}\right)=D\left(x_{1}\right) x_{2}+x_{1} D\left(x_{2}\right) \text {, for all } x_{1}, x_{2} \in \mathbb{Q}\left[x_{0}, x_{1}, x_{2}, \ldots, x_{n}\right] .
$$

A derivation $D$ is called locally nilpotent if for every $f \in \mathbb{Q}\left[x_{0}, x_{1}, x_{2}, \ldots, x_{n}\right]$ there is an $n \in \mathbb{N}$ such that $D^{n}(f)=0$. Any derivation $D$ is completely determined by the elements $D\left(x_{i}\right)$. A derivation $D$ is called linear if $D\left(x_{i}\right)$ is a linear form. A linear locally nilpotent derivation is called a Weitzenböck derivation. A derivation $D$ is called the triangular if $D\left(x_{i}\right) \in$ $\mathbb{Q}\left[x_{0}, \ldots, x_{i-1}\right]$. Any triangular derivation is locally nilpotent.

The subalgebra

$$
\text { ker } D:=\left\{f \in \mathbb{Q}\left[x_{0}, x_{1}, x_{2}, \ldots, x_{n}\right] \mid D(f)=0\right\},
$$

is called the kernel of derivation $D$.

For arbitrary locally nilpotent derivation $D$ the following statement holds:

Theorem 2.1. Suppose that there exists a polynomials $h$ such that $D(h) \neq 0$ but $D^{2}(h)=0$. Then

$$
\operatorname{ker} D=\mathbb{Q}\left[\sigma\left(x_{0}\right), \sigma\left(x_{1}\right), \ldots, \sigma\left(x_{n}\right)\right]\left[D(h)^{-1}\right] \cap \mathbb{Q}\left[x_{0}, x_{1}, \ldots, x_{n}\right] \text {, }
$$

where $\sigma$ is the Diximier map

$$
\sigma\left(x_{i}\right)=\sum_{k=0}^{\infty} D^{k}\left(x_{i}\right) \frac{\lambda^{k}}{k !}, \lambda=-\frac{h}{D(h)}, D(\lambda)=-1 .
$$

The element $\lambda$ is called a slice of the locally nilpotent derivation $D$. The proof one may find in [5] and [6]. 
2.2. Derivatives of the Kravchuk polynomials. The ordinary generating function for the Kravchuk polynomials has the form

$$
\sum_{i=0}^{\infty} K_{i}(x, a) z^{i}=(1+z)^{a}\left(\frac{1-z}{1+z}\right)^{x}
$$

Differentiating with respect to $x$ we get the generation function for the derivative $\frac{d}{d x} K_{i}(x, a)$ :

$$
\sum_{i=0}^{\infty} \frac{d}{d x} K_{i}(x, a) z^{i}=(1+z)^{a}\left(\frac{1-z}{1+z}\right)^{x} \ln \left(\frac{1-z}{1+z}\right)=\left(\sum_{i=0}^{\infty} K_{i}(x, a) z^{i}\right) \ln \left(\frac{1-z}{1+z}\right) .
$$

Taking into account

$$
\begin{gathered}
\ln \left(\frac{1-z}{1+z}\right)=\ln (1-z)-\ln (1+z)=-\sum_{i=1}^{\infty} \frac{z^{i}}{i}+\sum_{i=1}^{\infty}(-1)^{i} \frac{z^{i}}{i}=\sum_{i=1}^{\infty}\left(-\frac{2}{2 i-1}\right) z^{2 i-1}= \\
=-2 \sum_{i=1}^{\infty} \frac{1-(-1)^{i}}{2 i} z^{i}
\end{gathered}
$$

we have

$$
\begin{gathered}
\frac{d}{d x} K_{n}(x, a)=\left[z^{n}\right]\left(\sum_{i=0}^{\infty} K_{i}(x, a) z^{i}\right)\left(-2 \sum_{i=1}^{\infty} \frac{1-(-1)^{i}}{2 i} z^{i}\right)= \\
=-2 \sum_{i=1}^{n} \frac{1-(-1)^{i}}{2 i} K_{n-i}(x, a) .
\end{gathered}
$$

In the paper [2] one may see another proof of the formula and in [3] is presented another expression for $\frac{d}{d x} K_{n}(x, a)$.

Differentiating the generating function with respect to $a$ we get

$$
\sum_{i=0}^{\infty} \frac{d}{d a} K_{i}(x, a) z^{i}=(1+z)^{a}\left(\frac{1-z}{1+z}\right)^{x} \ln (1+z)=\left(\sum_{i=1}^{\infty} K_{i}(x, a) z^{i}\right)\left(\sum_{i=1}^{\infty}(-1)^{i+1} \frac{z^{i}}{i}\right) .
$$

In the same way, by using that

$$
\sum_{i=0}^{\infty} \frac{d}{d a} K_{i}(x, a) z^{i}=\left(\sum_{i=0}^{\infty} K_{i}(x, a) z^{i}\right) \ln (1+z),
$$

we obtain an expression for the derivatives with respect to $a$ :

$$
\frac{d}{d a} K_{n}(x, a)=\sum_{i=0}^{n-1} \frac{(-1)^{n+1+i}}{n-i} K_{i}(x, a) .
$$

2.3. Kravchuk derivations. The expressions for $\frac{d}{d x} K_{n}(x, a)$ and $\frac{d}{d a} K_{n}(x, a)$ motivate the following definition 
Definition 1. Derivations of $\mathbb{Q}\left[x_{0}, x_{1}, x_{2}, \ldots, x_{n}\right]$ defined by

$$
\begin{aligned}
& D_{\mathcal{K}_{1}}\left(x_{0}\right)=0, D_{\mathcal{K}}\left(x_{n}\right)=\sum_{i=1}^{n} \frac{1-(-1)^{i}}{2 i} x_{n-i}, \\
& D_{\mathcal{K}_{2}}\left(x_{0}\right)=0, D_{\mathcal{K}_{2}}\left(x_{n}\right)=\sum_{i=0}^{n-1} \frac{(-1)^{n+1+i}}{n-i} x_{i}, n=1,2, \ldots, n, \ldots,
\end{aligned}
$$

are called the first Kravchuk derivation and the second Kravchuk derivation respectively.

We have

$$
\begin{aligned}
D_{\mathcal{K}_{1}}\left(x_{0}\right) & =0, & D_{\mathcal{K}_{2}}\left(x_{0}\right) & =0, \\
D_{\mathcal{K}_{1}}\left(x_{1}\right) & =x_{0}, & D_{\mathcal{K}_{2}}\left(x_{1}\right) & =x_{0}, \\
D_{\mathcal{K}_{1}}\left(x_{2}\right) & =x_{1}, & D_{\mathcal{K}_{2}}\left(x_{2}\right) & =-\frac{1}{2} x_{0}+x_{1}, \\
D_{\mathcal{K}_{1}}\left(x_{3}\right) & =\frac{1}{3} x_{0}+x_{2}, & D_{\mathcal{K}_{2}}\left(x_{3}\right) & =\frac{1}{3} x_{0}-\frac{1}{2} x_{1}+x_{2}, \\
D_{\mathcal{K}_{1}}\left(x_{4}\right) & =\frac{1}{3} x_{1}+x_{3}, & D_{\mathcal{K}_{2}}\left(x_{4}\right) & =-\frac{1}{4} x_{0}+\frac{1}{3} x_{1}-\frac{1}{2} x_{2}+x_{3}, \\
D_{\mathcal{K}_{1}}\left(x_{5}\right) & =\frac{1}{5} x_{0}+\frac{1}{3} x_{2}+x_{4}, & D_{\mathcal{K}_{2}}\left(x_{5}\right) & =\frac{1}{5} x_{0}-\frac{1}{4} x_{1}+\frac{1}{3} x_{2}-\frac{1}{2} x_{3}+x_{4}, \\
D_{\mathcal{K}_{1}}\left(x_{6}\right) & =\frac{1}{5} x_{1}+\frac{1}{3} x_{3}+x_{5}, & D_{\mathcal{K}_{2}}\left(x_{6}\right) & =-\frac{1}{6} x_{0}+\frac{1}{5} x_{1}-\frac{1}{4} x_{2}+\frac{1}{3} x_{3}-\frac{1}{2} x_{4}+x_{5} .
\end{aligned}
$$

We define the substitution homomorphism $\varphi_{\mathcal{K}}: \mathbb{Q}\left[x_{0}, x_{1}, \ldots, x_{n}\right] \rightarrow \mathbb{Q}[x]$ by $\varphi_{\mathcal{K}}\left(x_{i}\right)=$ $\left.K_{i}(x, a)\right)$. Put

$$
\begin{aligned}
& \operatorname{ker} \varphi_{\mathcal{K}}:=\left\{P\left(x_{0}, x_{1}, \ldots, x_{n}\right) \in \mathbb{Q}\left[x_{0}, x_{1}, \ldots, x_{n}\right] \mid \varphi_{\mathcal{K}}\left(P\left(x_{0}, x_{1}, \ldots, x_{n}\right)\right) \in \mathbb{Q}\right\}, \\
& \operatorname{ker} \varphi_{\mathcal{K}_{1}}:=\left\{P\left(x_{0}, x_{1}, \ldots, x_{n}\right) \in \mathbb{Q}\left[x_{0}, x_{1}, \ldots, x_{n}\right] \mid \varphi_{\mathcal{K}}\left(P\left(x_{0}, x_{1}, \ldots, x_{n}\right)\right) \in \mathbb{Q}[a]\right\}, \\
& \operatorname{ker} \varphi_{\mathcal{K}_{2}}:=\left\{P\left(x_{0}, x_{1}, \ldots, x_{n}\right) \in \mathbb{Q}\left[x_{0}, x_{1}, \ldots, x_{n}\right] \mid \varphi_{\mathcal{K}}\left(P\left(x_{0}, x_{1}, \ldots, x_{n}\right)\right) \in \mathbb{Q}[x]\right\} .
\end{aligned}
$$

Any element of ker $\varphi_{\mathcal{K}_{1}}$ and ker $\varphi_{\mathcal{K}_{2}}$ gives a polynomial identity for the Kravchuk polynomials respectively. Put

$$
\begin{aligned}
& \operatorname{ker} \mathcal{D}_{\mathcal{K}_{1}}:=\left\{S \in \mathbb{Q}\left[x_{0}, x_{1}, \ldots, x_{n}\right] \mid \mathcal{D}_{\mathcal{K}_{1}}(S)=0\right\}, \\
& \operatorname{ker} \mathcal{D}_{\mathcal{K}_{2}}:=\left\{S \in \mathbb{Q}\left[x_{0}, x_{1}, \ldots, x_{n}\right] \mid \mathcal{D}_{\mathcal{K}_{2}}(S)=0\right\}
\end{aligned}
$$

It is easy to see that $\varphi_{\mathcal{K}} \mathcal{D}_{\mathcal{K}_{1}}=\frac{d}{d x} \varphi_{\mathcal{K}}$ and $\varphi_{\mathcal{K}} \mathcal{D}_{\mathcal{K}_{2}}=\frac{d}{d a} \varphi_{\mathcal{K}}$. It follows that $\varphi_{\mathcal{K}}\left(\operatorname{ker} \mathcal{D}_{\mathcal{K}_{1}}\right) \subset$ $\operatorname{ker}_{1} \varphi_{\mathcal{K}}$ and $\varphi_{\mathcal{K}}\left(\operatorname{ker} \mathcal{D}_{\mathcal{K}_{2}}\right) \subset \operatorname{ker}_{2} \varphi_{\mathcal{K}}$.

We have thus proved the following theorem.

Theorem 2.2. Let $P\left(x_{0}, x_{1}, \ldots, x_{n}\right)$ be a polynomial.

(i) If $\mathcal{D}_{\mathcal{K}_{1}}\left(P\left(x_{0}, x_{1}, \ldots, x_{n}\right)\right)=0$ then $P\left(K_{0}(x, a), K_{1}(x, a), \ldots, K_{n}(x, a)\right) \in \mathbb{Q}[a]$;

(ii) if $\mathcal{D}_{\mathcal{K}_{2}}\left(P\left(x_{0}, x_{1}, \ldots, x_{n}\right)\right)=0$ then $P\left(K_{0}(x, a), K_{1}(x, a), \ldots, K_{n}(x, a)\right) \in \mathbb{Q}[x]$.

Note that $\varphi_{\mathcal{K}}\left(\operatorname{ker} \mathcal{D}_{\mathcal{K}_{1}}\right) \neq \operatorname{ker} \varphi_{\mathcal{K}}$ and $\varphi_{\mathcal{K}}\left(\operatorname{ker} \mathcal{D}_{\mathcal{K}_{2}}\right) \neq \operatorname{ker} \varphi_{\mathcal{K}}$. In fact, we have

$$
\varphi_{\mathcal{K}}\left(x_{3} x_{1}^{2}-2 x_{2} x_{3} x_{0}-x_{1} x_{4} x_{0}-3 x_{3} x_{0}^{2}+5 x_{5} x_{0}^{2}\right)=0
$$


but $x_{3} x_{1}^{2}-2 x_{2} x_{3} x_{0}-x_{1} x_{4} x_{0}-3 x_{3} x_{0}^{2}+5 x_{5} x_{0}^{2} \notin \operatorname{ker} \mathcal{D}_{\mathcal{K}_{1,2}}$.

2.4. The kernel of the first Kravchuk derivation. It is obviously that that Kravchuk derivations are triangular and thus locally nilpotent. Thus to find its kernels we may use the Theorem 2.1.

Let us construct the Diximier map for the first Kravchuk derivation. For this purpose, we derive first a close expression for the powers $D_{\mathcal{K}_{1}}^{k}\left(x_{n}\right)$. Differentiating $k$ times the generating function with respect to $x$ we get

$$
\sum_{i=1}^{\infty} K_{i}(x, a) z^{i}=\ln \left(\frac{1-z}{1+z}\right)
$$

and taking into account

$$
\sum_{i=1}^{\infty} \frac{d}{d x} K_{i}(x, a) z^{i}=\left(\sum_{i=0}^{\infty} K_{i}(x, a) z^{i}\right) \ln \left(\frac{1-z}{1+z}\right),
$$

we get

$$
\sum_{i=k}^{\infty} \frac{d^{k}}{d x^{k}} K_{i}(x, a) z^{i}=\left(\sum_{i=0}^{\infty} K_{i}(x, a) z^{i}\right)\left(\ln \left(\frac{1+z}{1-z}\right)\right)^{k}
$$

By using the expansion

$$
\left(\ln \left(\frac{1+z}{1-z}\right)\right)^{k}=\left(\sum_{i=k}^{\infty} S^{(k)}(i) z^{i}\right)
$$

where

$$
S^{(k)}(n)=\sum_{m=k}^{n}\left(\begin{array}{c}
n-1 \\
m-1
\end{array}\right) \frac{2^{m} k !}{m !} s(m, k),
$$

and $s(m, k)$ are the Stirling numbers of the first kind. Then

$$
\sum_{i=k}^{\infty} \frac{d^{k}}{d x^{k}} K_{i}(x, a) z^{i}=\sum_{n=k}^{\infty}\left(\sum_{i=0}^{n-k} K_{i}(x, a) S^{(k)}(n-i)\right) z^{n} .
$$

Thus

$$
\mathcal{D}_{\mathcal{K}_{1}}^{k}\left(x_{n}\right)=\sum_{i=0}^{n-k} x_{i} S^{(k)}(n-i)
$$

Now we may find the Diximier map:

$$
\sigma\left(x_{n}\right)=\sum_{k=0}^{n} D_{\mathcal{K}_{1}}^{k}\left(x_{n}\right) \frac{\lambda^{k}}{k !}=\sum_{k=0}^{n} \frac{\lambda^{k}}{k !} \sum_{i=0}^{n-k} x_{i} S^{(k)}(n-i)=\sum_{i=0}^{n} x_{i} \sum_{k=0}^{n-i} \frac{\lambda^{k}}{k !} S^{(k)}(n-i) .
$$


Replacing $\lambda$ by $-\frac{x_{1}}{x_{0}}$, we obtain, after simplifying:

$$
\begin{gathered}
\sigma\left(x_{n}\right)=\sum_{i=0}^{n} x_{i} \sum_{k=0}^{n-i} \frac{(-1)^{k}}{k !}\left(\frac{x_{1}}{x_{0}}\right)^{k} S^{(k)}(n-i)= \\
=x_{0} \frac{(-1)^{n}}{n !}\left(\frac{x_{1}}{x_{0}}\right)^{n}+x_{1} \frac{(-1)^{n-1}}{(n-1) !}\left(\frac{x_{1}}{x_{0}}\right)^{n-1}+\sum_{i=0}^{1} x_{i} \sum_{k=0}^{n-i-1} \frac{(-1)^{k}}{k !}\left(\frac{x_{1}}{x_{0}}\right)^{k} S^{(k)}(n-i)+ \\
+\sum_{i=2}^{n} x_{i} \sum_{k=0}^{n-i} \frac{(-1)^{k}}{k !}\left(\frac{x_{1}}{x_{0}}\right)^{k} S^{(k)}(n-i)=\frac{(-1)^{n-1}}{n(n-2) !} \frac{x_{1}^{n}}{x_{0}^{n-1}}+\sum_{i=0}^{1} x_{i} \sum_{k=0}^{n-i-1} \frac{(-1)^{k}}{k !}\left(\frac{x_{1}}{x_{0}}\right)^{k} S^{(k)}(n-i)+ \\
+\sum_{i=2}^{n} x_{i} \sum_{k=0}^{n-i} \frac{(-1)^{k}}{k !}\left(\frac{x_{1}}{x_{0}}\right)^{k} S^{(k)}(n-i) .
\end{gathered}
$$

The polynomials

$$
\begin{array}{r}
C_{n}:=n(n-2) ! x_{0}^{n-1} \sigma\left(x_{n}\right)=(-1)^{n-1} x_{1}^{n}+n(n-2) ! \sum_{i=0}^{1} x_{i} \sum_{k=0}^{n-i-1} \frac{(-1)^{k}}{k !} x_{0}^{n-1-k} x_{1}^{k} S^{(k)}(n-i)+ \\
+n(n-2) ! \sum_{i=2}^{n} x_{i} \sum_{k=0}^{n-i} \frac{(-1)^{k}}{k !} x_{0}^{n-1-k} x_{1}^{k} S^{(k)}(n-i), n>1,
\end{array}
$$

belong to the kernel ker $\mathcal{D}_{\mathcal{K}_{1}}$. We call them the Cayley elements of the locally nilpotent derivation $\mathcal{D}_{\mathcal{K}_{1}}$. The first few Cayley elements are shown below:

$C_{2}=2 x_{2} x_{0}-x_{1}^{2}$,

$C_{3}=3 x_{3} x_{0}^{2}-x_{1} x_{0}^{2}-3 x_{1} x_{0} x_{2}+x_{1}^{3}$,

$C_{4}=8 x_{4} x_{0}{ }^{3}-8 x_{1} x_{0}{ }^{2} x_{3}+4 x_{1}{ }^{2} x_{0} x_{2}-x_{1}{ }^{4}$,

$C_{5}=30 x_{5} x_{0}^{4}-30 x_{1} x_{0}{ }^{3} x_{4}-10 x_{1} x_{0}{ }^{3} x_{2}-6 x_{1} x_{0}{ }^{4}+5 x_{1}{ }^{3} x_{0}{ }^{2}+15 x_{1}{ }^{2} x_{0}{ }^{2} x_{3}-5 x_{1}{ }^{3} x_{0} x_{2}+x_{1}{ }^{5}$,

$C_{6}=144 x_{6} x_{0}{ }^{5}+8 x_{1}{ }^{2} x_{0}{ }^{4}-48 x_{1} x_{0}{ }^{4} x_{3}-144 x_{1} x_{0}{ }^{4} x_{5}+48 x_{1}{ }^{2} x_{0}{ }^{3} x_{2}+72 x_{1}{ }^{2} x_{0}{ }^{3} x_{4}-16 x_{1}{ }^{4} x_{0}{ }^{2}-$

$-24 x_{1}{ }^{3} x_{0}{ }^{2} x_{3}+6 x_{1}{ }^{4} x_{0} x_{2}-x_{1}{ }^{6}$.

Theorem 2.1 implies

\section{Theorem 2.3.}

$$
\operatorname{ker} \mathcal{D}_{\mathcal{K}_{1}}=\mathbb{Q}\left[x_{0}, x_{1}, C_{3}, C_{4}, \ldots, C_{n}\right]\left[x_{1}^{-1}\right] \cap \mathbb{Q}\left[x_{0}, x_{1}, \ldots, x_{n}\right] .
$$

Thus, we obtain a description of the kernel of the Kravchuk derivation.

To get an identity for the Kravchuk polynomials we should find $\varphi_{F}\left(C_{n}\right)$.

We have

$$
\varphi_{\mathcal{K}}\left(C_{n}\right)=\varphi_{K}\left(\sigma\left(x_{n}\right)\right)=\sum_{i=0}^{n} K_{i} \sum_{k=0}^{n-i} \frac{(-1)^{k}}{k !} K_{1}^{k} S^{(k)}(n-i) .
$$


By Theorem 2.2 the right side is a function of $a$. We have

$$
\begin{aligned}
\varphi_{\mathcal{K}}\left(C_{2}\right) & =-a \\
\varphi_{\mathcal{K}}\left(C_{3}\right) & =0 \\
\varphi_{\mathcal{K}}\left(C_{4}\right) & =a(a-2) \\
\varphi_{\mathcal{K}}\left(C_{5}\right) & =0 \\
\varphi_{\mathcal{K}}\left(C_{6}\right) & =-3 a(a-2)(a-4)
\end{aligned}
$$

We would like to propose the following conjecture for the Kravchuk polynomials: Conjecture 1.

$$
\begin{gathered}
\sum_{i=0}^{n} K_{i}(x, a) \sum_{k=0}^{n-i} \frac{(-1)^{k}}{k !} K_{1}(x, a)^{k} S^{(k)}(n-i)= \\
=\left\{\begin{array}{ll}
0, & n \text { odd }, \\
(-1)^{m}(2 m-1) ! ! a(a-2)(a-4) \ldots(a-2(m-1)), & n=2 m .
\end{array},\right.
\end{gathered}
$$

where

$$
S^{(k)}(n)=\sum_{m=k}^{n}\left(\begin{array}{c}
n-1 \\
m-1
\end{array}\right) \frac{2^{m} k !}{m !} s(m, k) .
$$

2.5. The kernel of the second Kravchuk derivation. In the same way, to derive a close expression for the powers $D_{\mathcal{K}_{2}}^{k}\left(x_{n}\right)$ we use the exponential generating function for the Stirling numbers of the first kind $s(n, k)$ :

$$
\sum_{n=k}^{\infty} s(n, k) \frac{z^{i}}{n !}=\frac{(\ln (1+z))^{k}}{k !} .
$$

We have

$$
\begin{gathered}
\sum_{i=k}^{\infty} \frac{d^{k}}{d a^{k}} K_{i}(x, a) z^{i}=\left(\sum_{i=0}^{\infty} K_{i}(x, a) z^{i}\right)(\ln (1+z))^{k}= \\
=\left(\sum_{i=0}^{\infty} K_{i}(x, a) z^{i}\right)\left(\sum_{i=k}^{\infty} \frac{k !}{n !} s(n, k) z^{i}\right)=\sum_{n=k}^{\infty}\left(\sum_{i=0}^{n-k} K_{i}(x, a) \frac{k !}{(n-i) !} s(n-i, k)\right) z^{n} .
\end{gathered}
$$

Thus

$$
\mathcal{D}_{\mathcal{K}_{2}}^{k}\left(x_{n}\right)=\sum_{i=0}^{n-k} x_{i} \frac{k !}{(n-i) !} s(n, k) .
$$

Since $D_{\mathcal{K}_{2}}\left(-\frac{x_{1}}{x_{0}}\right)=-1$ we put $\lambda=-\frac{x_{1}}{x_{0}}$. Now we may find the Diximier map:

$$
\begin{gathered}
\sigma\left(x_{n}\right)=\sum_{k=0}^{n} D_{\mathcal{K}_{2}}^{k}\left(x_{n}\right) \frac{\lambda^{k}}{k !}= \\
=\sum_{k=0}^{n} \frac{\lambda^{k}}{k !} \sum_{i=0}^{n-k} x_{i} \frac{k !}{(n-i) !} s(n-i, k)=\sum_{i=0}^{n} x_{i} \sum_{k=0}^{n-i} \frac{\lambda^{k}}{(n-i) !} s(n-i, k) .
\end{gathered}
$$


Replacing $\lambda$ by $-\frac{x_{1}}{x_{0}}$, we obtain:

$$
\sigma\left(x_{n}\right)=\sum_{i=0}^{n} x_{i} \sum_{k=0}^{n-i} \frac{(-1)^{k}}{(n-i) !} \frac{x_{1}^{k}}{x_{0}^{k}} s(n-i, k) .
$$

The first few Cayley elements are shown below:

$$
\begin{aligned}
\sigma\left(x_{2}\right) & =\frac{1}{2} \frac{x_{1} x_{0}-x_{1}^{2}+2 x_{2} x_{0}}{x_{0}}, \\
\sigma\left(x_{3}\right) & =-\frac{1}{3} \frac{x_{1} x_{0}^{2}-x_{1}^{3}+3 x_{2} x_{1} x_{0}-3 x_{3} x_{0}^{2}}{x_{0}^{2}}, \\
\sigma\left(x_{4}\right) & =\frac{1}{8} \frac{2 x_{1} x_{0}^{3}+x_{1}^{2} x_{0}^{2}-2 x_{1}^{3} x_{0}-x_{1}^{4}+4 x_{2} x_{1} x_{0}^{2}+4 x_{2} x_{1}^{2} x_{0}-8 x_{3} x_{1} x_{0}^{2}+8 x_{4} x_{0}{ }^{3}}{x_{0}{ }^{3}} .
\end{aligned}
$$

By using the substantial homomorphism $\varphi_{\mathcal{K}}$ we get:

$$
\begin{aligned}
& \varphi_{\mathcal{K}}\left(\sigma\left(x_{2}\right)\right)=\frac{1}{2}\left(K_{1}(x, a) K_{0}(x, a)-K_{1}(x, a)^{2}+2 K_{2}(x, a) K_{0}(x, a)\right)=-x, \\
& \varphi_{\mathcal{K}}\left(\sigma\left(x_{3}\right)\right)=-\frac{1}{3}\left(K_{1}(x, a) K_{0}(x, a)^{2}-K_{1}(x, a)^{3}+3 K_{2}(x, a) K_{1}(x, a) K_{0}(x, a)-\right. \\
& \left.-3 K_{3}(x, a) K_{0}(x, a)^{2}\right)=0, \\
& \varphi_{\mathcal{K}}\left(\sigma\left(x_{4}\right)\right)=\frac{1}{8}\left(2 K_{1}(x, a) K_{0}(x, a)^{3}+K_{1}(x, a)^{2} K_{0}(x, a)^{2}-2 K_{1}(x, a)^{3} K_{0}(x, a)-\right. \\
& K_{1}(x, a)^{4}+4 K_{2}(x, a) K_{1}(x, a) K_{0}(x, a)^{2}+4 K_{2}(x, a) K_{1}(x, a)^{2} K_{0}(x, a)- \\
& \left.-8 K_{3}(x, a) K_{1}(x, a) K_{0}(x, a)^{2}+8 K_{4}(x, a) K_{0}(x, a)^{3}\right)=\frac{1}{2}(x-1) x, \\
& \varphi_{\mathcal{K}}\left(\sigma\left(x_{5}\right)\right)=0, \\
& \varphi_{\mathcal{K}}\left(\sigma\left(x_{6}\right)\right)=-\frac{1}{6} x(x-1)(x-2),
\end{aligned}
$$

For the general case we propose the following conjecture:

\section{Conjecture 2.}

$$
\sum_{i=0}^{n} K_{i}(x, a) \sum_{k=0}^{n-i} \frac{(-1)^{k}}{(n-i) !} K_{1}(x, a)^{k} s(n-i, k)= \begin{cases}0, & n \text { odd }, \\
(-1)^{m}\left(\begin{array}{c}
x \\
m
\end{array}\right), & n=2 m .\end{cases}
$$

\section{ApPeL-KRAVChUK INTERTWINING MAPS}

3.1. The first Kravchuk derivation. Denote by $\psi_{A K_{1}}$ an $\left(\mathcal{D}, \mathcal{D}_{\mathcal{K}_{1}}\right)$ intertwining map. Suppose it has the form :

$$
\psi_{A K_{1}}\left(x_{0}\right)=x_{0}, \psi_{A K_{1}}\left(x_{n}\right)=\sum_{i=1}^{n} T(n, i) x_{i} .
$$

After direct calculations we obtain

$$
\begin{aligned}
& \psi_{A K_{1}}\left(x_{0}\right)=x_{0}, \psi_{A K_{1}}\left(x_{1}\right)=x_{1}, \psi_{A K_{1}}\left(x_{2}\right)=2 x_{2}, \\
& \psi_{A K_{1}}\left(x_{3}\right)=-2 x_{1}+6 x_{3}, \psi_{A K_{1}}\left(x_{4}\right)=-16 x_{2}+24 x_{4}, \\
& \psi_{A K_{1}}\left(x_{5}\right)=16 x_{1}-120 x_{3}+120 x_{5}, \psi_{A K_{1}}\left(x_{6}\right)=272 x_{2}-960 x_{4}+720 x_{6} .
\end{aligned}
$$


Let us prove the following statement

Theorem 3.1. The numbers $T(n, i)$ are the following explicit form

$$
T(n, i)=\sum_{j=i}^{n}(-1)^{j-i} 2^{n-j} j ! S(n, j)\left(\begin{array}{c}
j-1 \\
i-1
\end{array}\right)
$$

where $S(n, j)$ are the Stirling numbers of the second kind.

Proof. To make sure that the initial conditions hold we calculate $T(0,0)=1, T(1,1)=$ $0, T(2,1)=0, T(2,2)=2$. By using the summation formula

$$
\sum_{i=1}^{n} a_{i} \sum_{j=1}^{i} b_{j} c_{i-j}=\sum_{i=0}^{n-1} c_{i} \sum_{j=i+1}^{n} a_{j} b_{j-i}
$$

we have

$$
\begin{aligned}
\left.\mathcal{D}_{\mathcal{K}_{1}} \psi_{A K_{1}}\left(x_{n}\right)\right)= & \sum_{i=1}^{n} T(n, i) \mathcal{D}_{\mathcal{K}_{1}}\left(x_{i}\right)=\sum_{i=1}^{n} T(n, i) \sum_{j=1}^{i} \frac{1-(-1)^{j}}{2 j} x_{i-j}= \\
& =\sum_{i=0}^{n-1} x_{i} \sum_{j=i+1}^{n} \frac{1-(-1)^{j-i}}{2(j-i)} T(n, j) .
\end{aligned}
$$

On the other hand

$$
D_{\mathcal{K}_{1}}\left(\psi_{A K_{1}}\left(x_{n}\right)\right)=\psi_{A K_{1}}\left(\mathcal{D}\left(x_{n}\right)\right)=n \psi_{A K_{1}}\left(x_{n-1}\right)=n \sum_{i=1}^{n-1} T(n-1, i) x_{i} .
$$

By equation the corresponding coefficients we obtain the recurrence relations for the sequences $T(n, i)$ :

$$
\sum_{j=i+1}^{n} \frac{1-(-1)^{j-i}}{2(j-i)} T(n, j)=n T(n-1, i), i=0,1, \ldots, n-1
$$

In order to prove the theorem, we need the following important lemma

Lemma 3.1. Let us consider two sequences $a_{n}$ and $A(n, k), A(n, k)=0, k>n$ with the generating functions:

$$
\sum_{n=k}^{\infty} A(n, k) \frac{z^{n}}{n !}=(f(z))^{k}, \sum_{n=0}^{\infty} a_{n} z^{k}=g(z) .
$$

Suppose that $g(f(z))=z$.

Then

$$
\sum_{j=i+1}^{n} a_{j-i} A(n, j)=\sum_{k=1}^{n-i} a_{n} A(n, k+i)=n A(n-1, i), i=0,1, \ldots, n-1 .
$$


Proof. Multiply the left side by $\frac{z^{n}}{n !}$ and sum on $n$ from $i$ to $\infty$ we get:

$$
\begin{aligned}
& \sum_{n=i}^{\infty} \sum_{j=i+1}^{n} a_{j-i} A(n, j) \frac{z^{n}}{n !}=\sum_{j=i+1}^{\infty} a_{j-i} \sum_{n=i}^{\infty} A(n, j) z^{n}=\sum_{j=i+1}^{\infty} a_{j-i}(f(z))^{j}=\sum_{k=1}^{\infty} a_{k}(f(z))^{i+k}= \\
& =(f(z))^{i} \sum_{k=1} a_{k}(f(z))^{k}=(f(z))^{i} g(f(z))=z(f(z))^{i}=z \sum_{n=0}^{\infty} A(n, i) \frac{z^{n}}{n !}=\sum_{n=1}^{\infty} n A(n-1, i) \frac{z^{n}}{n !} .
\end{aligned}
$$

Since

$$
\sum_{n=0}^{\infty} \sum_{j=i+1}^{n} a_{j-i} A(n, j) \frac{z^{n}}{n !}=\sum_{n=0}^{\infty}\left(\sum_{j=i+1}^{n} a_{j-i} A(n, j)\right) \frac{z^{n}}{n !},
$$

then by equation the corresponding coefficients we get the identities

$$
\sum_{j=i+1}^{n} a_{j-i} A(n, j)=\sum_{k=1}^{n-i} a_{n} A(n, k+i)=n A(n-1, i),
$$

as required.

Now, let us find the exponential generating function for the numbers

$$
T(n, i)=\sum_{j=i}^{n}(-1)^{j-i} 2^{n-j} j ! S(n, j)\left(\begin{array}{l}
j-1 \\
i-1
\end{array}\right) .
$$

Multiply the left and right sides by $\frac{z^{n}}{n !}$ and sum on $n$ from $i$ to $\infty$. Taking into account that

$$
\sum_{n=i}^{\infty} S(n, j) \frac{(z)^{n}}{n !}=\frac{\left(e^{z}-1\right)^{j}}{j !}
$$

we obtain

$$
\begin{gathered}
\sum_{n=i}^{\infty} T(n, i) \frac{z^{n}}{n !}=\sum_{n=i}^{\infty} \sum_{j=i}^{\infty}(-1)^{j-i} 2^{n-j} j !\left(\begin{array}{c}
j-1 \\
i-1
\end{array}\right) S(n, j) \frac{z^{n}}{n !}= \\
=\sum_{j=i}^{\infty} \frac{(-1)^{j-i}}{2^{j}} j !\left(\begin{array}{c}
j-1 \\
i-1
\end{array}\right) \sum_{n=i}^{\infty} S(n, j) \frac{(2 z)^{n}}{n !}=\sum_{j=i}^{\infty} \frac{(-1)^{j-i}}{2^{j}}\left(\begin{array}{c}
j-1 \\
i-1
\end{array}\right)\left(e^{2 z}-1\right)^{j} .
\end{gathered}
$$

By using the expansion

$$
\sum_{j=i}^{\infty} \frac{(-1)^{j-i}}{2^{j}}\left(\begin{array}{c}
j-1 \\
i-1
\end{array}\right) z^{j}=\left(\frac{z}{z+2}\right)^{i}
$$

we get

$$
\sum_{n=i}^{\infty} T(n, i) \frac{z^{n}}{n !}=\left(\frac{e^{2 z}-1}{e^{2 z}+1}\right)^{i}
$$

Consider the sequence

$$
a_{n}=\frac{1-(-1)^{n}}{2 n} .
$$


Its ordinary generating function has the form

$$
g(z)=\sum_{n=1}^{\infty} a_{n} z^{k}=\frac{1}{2} \ln \left(\frac{1+z}{1-z}\right) .
$$

Put $f(z)=\frac{e^{2 z}-1}{e^{2 z}+1}$. It is easy to see that $g(f(z))=z$. Therefore, by Lemma 3.1, we get that the numbers $T(n, i)$ are solutions of the system of recurrence equations

$$
\sum_{j=i+1}^{n} \frac{1-(-1)^{j-i}}{2(j-i)} T(n, j)=n T(n-1, i), i=0,1, \ldots, n-1,
$$

which is what had to be proved.

3.2. The second Kravchuk derivation. Denote by $\psi_{A K_{2}}$ an $\left(\mathcal{D}, \mathcal{D}_{\mathcal{K}_{2}}\right)$ intertwining map. Suppose it has the form :

$$
\psi_{A K_{2}}\left(x_{0}\right)=x_{0}, \psi_{A K_{2}}\left(x_{n}\right)=\sum_{i=1}^{n} B(n, i) x_{i}, n>0
$$

We have

$$
\begin{aligned}
& \psi_{A K_{2}}\left(x_{0}\right)=x_{0}, \psi_{A K_{2}}\left(x_{1}\right)=x_{1}, \psi_{A K_{2}}\left(x_{2}\right)=x_{1}+2 x_{2}, \\
& \psi_{A K_{2}}\left(x_{3}\right)=x_{1}+6 x_{2}+6 x_{3}, \psi_{A K_{2}}\left(x_{4}\right)=x_{1}+14 x_{2}+36 x_{3}+24 x_{4}, \\
& \psi_{A K_{2}}\left(x_{5}\right)=x_{1}+30 x_{2}+150 x_{3}+240 x_{4}+120 x_{5}, \\
& \psi_{A K_{2}}\left(x_{6}\right)=x_{1}+62 x_{2}+540 x_{3}+1560 x_{4}+1800 x_{5}+720 x_{6} .
\end{aligned}
$$

Let us prove the following statement

\section{Lemma 3.2.}

$$
B(n, k)=k ! S(n, k) .
$$

Proof. We have $B(0,0)=1, B(1,1)=0, B(2,1)=1, B(2,2)=2$, thus the initial conditions hold. By using the summation formula

$$
\sum_{i=1}^{n} a_{i} \sum_{j=0}^{i-1} b_{i, j} c_{j}=\sum_{i=0}^{n-1} c_{i} \sum_{j=i+1}^{n} a_{j} b_{j, i}
$$

we have

$$
\begin{aligned}
D_{\mathcal{K}_{2}}\left(\psi_{A K_{2}}\left(x_{n}\right)\right)= & D_{\mathcal{K}_{2}}\left(\sum_{i=1}^{n} B(n, i) x_{i}\right)=\sum_{i=1}^{n} B(n, i) \sum_{i=0}^{i-1} \frac{(-1)^{i+1-j}}{i-j} x_{j}= \\
= & \sum_{i=0}^{n-1} x_{i} \sum_{j=i+1}^{n} \frac{(-1)^{j+1-i}}{j-i} B(n, j) .
\end{aligned}
$$

On the other hand

$$
D_{\mathcal{K}_{2}}\left(\psi_{A K_{2}}\left(x_{n}\right)\right)=\psi_{A K_{2}}\left(\mathcal{D}\left(x_{n}\right)\right)=n \psi_{A K_{2}}\left(x_{n-1}\right)=n \sum_{i=1}^{n-1} B(n-1, i) x_{i} .
$$


By equation the corresponding coefficients we obtain that the numbers $B(n, k)$ satisfy the following system of recurrence relations:

$$
\sum_{j=i+1}^{n} \frac{(-1)^{j+1-i}}{j-i} B(n, j)=n B(n-1, i), i=0,1, \ldots, n-1 .
$$

To prove that the numbers $B(n, k)=k ! S(n, k)$ are the solutions of the system we use the Lemma 3.1. Under conditions of this lemma we put

$$
a_{i}=\frac{(-1)^{i+1}}{i}, A(n, i)=B(n, i) .
$$

Then

$$
g(x)=\sum_{i=1}^{\infty} a_{i} z^{n}=\sum_{i=1}^{\infty} \frac{(-1)^{i+1}}{i} z^{n}=\ln (1+z) .
$$

Also use the exponential generating function for the Stirling numbers of the second kind

$$
\sum_{n=1}^{\infty} j ! S(n, j) \frac{x^{n}}{n !}=\left(e^{x}-1\right)^{j} .
$$

It follows that $f(z)=e^{x}-1$. Since $g(f(z))=z$, then all conditions of the Lemma 3.1 hold. Thus the numbers $B(n, i)$ are the solutions of the system of recurrence equations:

$$
\sum_{j=i+1}^{n} \frac{(-1)^{j+1-i}}{j-i} B(n, j)=n B(n-1, i), i=0,1, \ldots, n-1 .
$$

3.3. Some identities. Having the explicit forms of the intertwining maps we may assist to any series of elements of kernels of the Weitzenböck derivations $\mathcal{D}$ an identity for the Kravchuk polynomials. A list of such series are presented in the paper [1]. For instance, the polynomials

$$
I_{n}=\frac{1}{2} \sum_{i=0}^{2 n}(-1)^{i}\left(\begin{array}{c}
2 n \\
i
\end{array}\right) x_{i} x_{n-i}
$$

belong to the kernel of the derivation $\mathcal{D}$, thus $\psi_{A K_{1}}\left(I_{n}\right)=\varphi_{1}(a), \psi_{A K_{2}}\left(I_{n}\right)=\varphi_{2}(a)$, where $\varphi_{1}$, and $\varphi_{2}$ are polynomials of one variable. It is a difficult problem to find the explicit form of the polynomials $\varphi_{1}$, and $\varphi_{2}$.

Another example of the element of the kernel $\mathcal{D}$ is the determinant of the Hankel matrix

$$
H_{n}:=\operatorname{det}\left(\psi_{A K_{1}}\left(x_{i+j-2}\right)\right)=\left|\begin{array}{ccccc}
x_{0} & x_{1} & x_{2} & \ldots & x_{n} \\
x_{1} & x_{2} & x_{3} & \ldots & x_{n+1} \\
\ldots \ldots & \ldots & \ldots \ldots \ldots \ldots \ldots \ldots \\
x_{n-1} & x_{n} & x_{n-1} & \ldots & x_{2 n-1} \\
x_{n} & x_{n+1} & x_{n+2} & \ldots & x_{2 n}
\end{array}\right| .
$$

For $\psi_{A K_{1}}\left(H_{n}\right), \psi_{A K_{2}}\left(H_{n}\right)$ we offer the conjecture: 


\section{Conjecture 3 .}

$$
\begin{aligned}
& \text { (i) } \psi_{A K_{1}}\left(H_{n}\right)=(-1)^{\frac{n(n+1)}{2}} \prod_{i=0}^{n-1} i ! \prod_{i=0}^{n-2}(i+a)^{n-1-i} \\
& \text { (ii) } \psi_{A K_{2}}\left(H_{n}\right)=(-1)^{\frac{n(n+1)}{2}} \prod_{i=0}^{n} 2^{i} i ! \prod_{i=0}^{n-2}(x-i)^{n-1-i} .
\end{aligned}
$$

\section{REFERENCES}

[1] L. Bedratyuk, Semi-invariants of binary forms and identities for Bernoulli, Euler and Hermite polynomials, Acta Arith., 151 (2012), 361-376

[2] Krasikov, I. and Litsyn, S., On integral zeros of Krawtchouk polynomials, Journal of Combinatorial Theory, Series A 74 (1) : 71- 99

[3] Koepf, Wolfram Identities for families of orthogonal polynomials and special functions, Integral Transform. Spec. Funct. 5 (1997), no. 1-2, 69-102.

[4] Glenn O. Treatise on theory of invariants.-Boston,1915.-312P.

[5] Nowicki A. Polynomial derivation and their Ring of Constants, -Torun:UMK,1994.-170p.

[6] van den Essen A. Polynomial automorphisms and the Jacobian conjecture.-Basel: Birkhäuser. -2000.-329 p.

Department of Applied Mathematics, Khmelnitsky National University, Khmelnitsky, InstyTUTSKA ,11, 29016, UKRAINE

E-mail address: leonid.uk@gmail.com 